\title{
Generic stability of the solution mapping for set-valued optimization problems
}

\author{
Xian-Jun Long ${ }^{*}$, Ying-Quan Huang and Li-Ping Tang
}

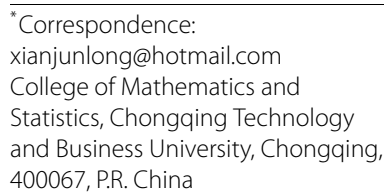

\begin{abstract}
In this paper, we consider the generic stability of the weakly efficient solution mapping for set-valued optimization problems. Firstly, we obtain the upper semicontinuity of the weakly efficient solution mapping for set-valued optimization problems. Secondly, we show that, in the sense of Baire category, most set-valued optimization problems are stable. Finally, we give sufficient conditions ensuring the existence of essential. Our results extend and improve the corresponding results of Song et al. (J. Optim. Theory Appl. 156:591-599, 2013).
\end{abstract}

MSC: 49J53; 90C29; 90C31

Keywords: generic stability; set-valued optimization problem; weakly efficient solution; essential; semicontinuity

\section{Introduction}

Set-valued optimization is a vibrant and expanding branch of applied mathematics that deals with optimization problems where the objective function is a set-valued map acting between abstract spaces. Set-valued optimization provides an important generalization and unification of scalar as well as vector optimization problems. Therefore, this relatively new discipline has justifiably attracted a great deal of attention in recent years (see [1-21]).

Stability is very interesting and important in optimization theory and applications. It may be understood as the solution set having some topological properties such as semicontinuity, well-posedness, essential stability and so on. Essential stability was firstly introduced by Fort [22] for the study of fixed points of a continuous mapping. Since then, essentiality was applied in many nonlinear problems such as KKM points, vector equilibrium problems and Nash equilibrium problems (see [22-27]). Recently, Xiang and Zhou [28] obtained the essential stability of efficient solution sets for continuous vector optimization problems. Very recently, Song et al. [29] generalized the results obtained by Xiang and Zhou [28] to a set-valued case. They obtained the essential stability of efficient solution sets for set-valued optimization problems with the only perturbation of the objective function in compact metric spaces.

In this paper, we consider the stability of a weakly efficient solution mapping for setvalued optimization problems with the perturbation of both the objective function and the constraint set in noncompact Banach spaces. In Section 2 we recall some basic definitions and some known results. In Section 3 we obtain the upper semicontinuity of the weakly

(c) 2015 Long et al. This article is distributed under the terms of the Creative Commons Attribution 4.0 International License (http://creativecommons.org/licenses/by/4.0/), which permits unrestricted use, distribution, and reproduction in any medium, provided you give appropriate credit to the original author(s) and the source, provide a link to the Creative Commons license, and indicate if changes were made. 
efficient solution mapping for set-valued optimization problems. Moreover, we show that, in the sense of Baire category, most set-valued optimization problems are stable. Finally, we give sufficient conditions ensuring the existence of essential. Our results extend and improve the corresponding results of Song et al. [29].

\section{Preliminaries}

Let $X$ and $Y$ be two topological vector spaces. Let $C \subset Y$ be a closed convex pointed cone with int $C \neq \emptyset$, where int $C$ denotes the interior of $C$. Let $A \subset Y$ be a nonempty subset. We denote by

$$
\operatorname{WMin} A:=\{y \in A:(A-y) \cap-\operatorname{int} C=\emptyset\}
$$

the set of weakly efficient elements of $A$ and by

$$
\operatorname{Min} A:=\{y \in A:(A-y) \cap-C=\{0\}\}
$$

the set of efficient elements of $A$.

Let $F: X \rightarrow 2^{Y}$ be a set-valued map, $K \subseteq X$ be a nonempty subset. We consider the following set-valued optimization problem (in short, SOP):

$$
\min _{C} F(x) \text { subject to } x \in K \text {. }
$$

We denote

$$
F(K)=\bigcup_{x \in K} F(x)
$$

Definition 2.1 A point $x_{0} \in K$ is said to be a weakly efficient (resp. an efficient) solution of problem (SOP) iff there exists $y_{0} \in F\left(x_{0}\right)$ such that $y_{0} \in \operatorname{WMin} F(K)$ (resp. $y_{0} \in \operatorname{Min} F(K)$ ).

Definition 2.2 [2] Let $G: X \rightarrow 2^{Y}$ be a set-valued map. $T$ is said to be

(1) upper semicontinuous at $x_{0} \in X$ if, for any open set $V$ containing $G\left(x_{0}\right)$, there exists a neighborhood $U\left(x_{0}\right)$ of $x_{0}$ such that $G(x) \subset V$ for all $x \in U\left(x_{0}\right)$; $G$ is said to be upper semicontinuous on $X$ if it is upper semicontinuous at each $x \in X$;

(2) lower semicontinuous at $x_{0} \in X$ if, for any open set $V$ with $G\left(x_{0}\right) \cap V \neq \emptyset$, there exists a neighborhood $U\left(x_{0}\right)$ of $x_{0}$ such that $G(x) \cap V \neq \emptyset$ for all $x \in U\left(x_{0}\right)$; $G$ is said to be lower semicontinuous on $X$ if it is lower semicontinuous at each $x \in X$;

(3) continuous on $X$ if it is both upper semicontinuous and lower semicontinuous on $X$;

(4) closed if $\operatorname{Graph}(G):=\{(x, y): x \in X, y \in G(x)\}$ is a closed set in $X \times Y$.

Lemma 2.1 [2] Let $G: X \rightarrow 2^{Y}$ be a set-valued map. If $G$ is upper semicontinuous and for any $x \in X, G(x)$ is a closed set, then $G$ is closed.

Definition 2.3 Let $(X, d)$ be a metric space and let $A, B$ be nonempty subsets of $X$. The Hausdorff distance $H(\cdot, \cdot)$ between $A$ and $B$ is defined by

$$
H(A, B)=\max \{e(A, B), e(B, A)\},
$$


where $e(A, B)=\sup _{a \in A} d(a, B)$ with $d(a, B)=\inf _{b \in B}\|a-b\|$. Let $\left\{A_{n}\right\}$ be a sequence of nonempty subsets of $X$. We say that $A_{n}$ converges to $A$ in the sense of Hausdorff distance (denoted by $A_{n} \rightarrow A$ ) if $H\left(A_{n}, A\right) \rightarrow 0$. It is easy to see that $e\left(A_{n}, A\right) \rightarrow 0$ if and only if $d\left(a_{n}, A\right) \rightarrow 0$ for all selection $a_{n} \in A_{n}$. For more details on this topic, we refer the readers to $[30,31]$.

Lemma 2.2 [32] Let $A$ and $A_{n}(n=1,2, \ldots)$ all be nonempty compact subsets of the Hausdorff topological space $X$ with $A_{n} \rightarrow A$. Then the following statements hold:

(i) $\bigcup_{n=1}^{+\infty} A_{n} \cup A$ is also a nonempty compact subset of $X$.

(ii) If $x_{n} \in A_{n}$ converging to $x$, then $x \in A$.

A topological space $X$ is said to be a Baire space if the following condition holds: given any countable collection $\left\{A_{n}\right\}_{n=1}^{+\infty}$ of the closed subsets of $X$ each of which has empty interior in $X$, their union $\cup A_{n}$ also has empty interior in $X$. A subset $G$ of $X$ is called residual if it contains a countable intersection of open dense subsets of $X$.

Lemma 2.3 (Baire category theorem) If $X$ is a compact Hausdorff space or a complete metric space, then $X$ is a Baire space.

Lemma 2.4 ([22], Theorem 2) Let $X$ be a Baire space, $Y$ be a metric space and $G: X \rightarrow 2^{Y}$ be upper semicontinuous with compact values. Then there exists a dense residual subset $Q$ of $X$ such that $G$ is lower semicontinuous at each $x \in Q$.

For convenience in the later presentation, denote by $K(X)$ and $K(Y)$ all nonempty compact subsets of $X$ and $Y$, respectively.

Lemma 2.5 [31] Let $(X, d)$ be a metric space and $H$ be Hausdorff distance on $X$. Then $(K(X), H)$ is complete if and only if $(X, d)$ is complete.

The next lemma is a special case of Lemma 2.4 in [24].

Lemma 2.6 Let $K$ be a nonempty compact subset of $X$ and $G: K \rightarrow 2^{Y}$ be a set-valued map with nonempty compact values. Then $G$ is continuous if and only if for any $x_{0} \in K$, $x \rightarrow x_{0}$ implies $G(x) \rightarrow G\left(x_{0}\right)$.

Lemma 2.7 [29] Let $F_{n} \rightarrow F, n=1,2, \ldots$, where $F_{n}, F: X \rightarrow 2^{Y}$ are continuous on $X$ and have nonempty compact values. If $y_{n} \in F_{n}\left(x_{n}\right), x_{n} \rightarrow x^{*}$ and $y_{n} \rightarrow y^{*}$, then $y^{*} \in F\left(x^{*}\right)$.

Lemma 2.8 Let $F_{n} \rightarrow F, n=1,2, \ldots$, where $F_{n}, F: X \rightarrow 2^{Y}$ are continuous on $X$ and have nonempty compact values. Then, for any $x \in X, y \in F(x), x_{n} \rightarrow x$, there exists $y_{n} \in F_{n}\left(x_{n}\right)$ such that $y_{n} \rightarrow y$.

Proof Since $F_{n} \rightarrow F, H\left(F_{n}(x), F(x)\right) \rightarrow 0$ for any $x \in X$. Note that

$$
H\left(F_{n}\left(x_{n}\right), F(x)\right) \leq H\left(F_{n}\left(x_{n}\right), F\left(x_{n}\right)\right)+H\left(F\left(x_{n}\right), F(x)\right) .
$$

By the continuity of $F$ and Lemma 2.6, $H\left(F_{n}\left(x_{n}\right), F(x)\right) \rightarrow 0$. Therefore, for any $y \in F(x)$, there exists $y_{n} \in F_{n}\left(x_{n}\right)$ such that $y_{n} \rightarrow y$. The proof is complete. 


\section{Main results}

Throughout this section, let $X$ and $Y$ be two real Banach spaces, $K$ be a nonempty subset of $X, C \subset Y$ be a closed convex pointed cone with int $C \neq \emptyset$.

The space $M$ of the problem (SOP) is defined by

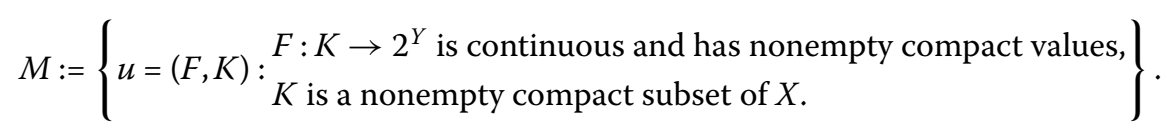

For any $u_{1}=\left(F_{1}, K_{1}\right), u_{2}=\left(F_{2}, K_{2}\right) \in M$, we define the metric $\rho$ as follows:

$$
\rho\left(u_{1}, u_{2}\right):=\sup _{x \in K} H_{F}\left(F_{1}(x), F_{2}(x)\right)+H_{K}\left(K_{1}, K_{2}\right)
$$

where $H_{F}, H_{K}$ are two Hausdorff distances on $Y$ and $X$, respectively.

Lemma 3.1 $(M, \rho)$ is a complete metric space.

Proof Clearly, $(M, \rho)$ is a metric space. We only need to show that $(M, \rho)$ is complete. Let $\left\{u_{n}\right\}$ be a Cauchy sequence of $M$, where $u_{n}=\left(F_{n}, K_{n}\right)$. Then, for any $\varepsilon>0$, there exists a positive integer $N_{1}$ such that

$$
\rho\left(u_{n}, u_{m}\right)<\frac{\varepsilon}{3} \quad \text { for all } m, n \geq N_{1} .
$$

It follows that for any $x \in K$,

$$
H_{F}\left(F_{n}(x), F_{m}(x)\right)<\frac{\varepsilon}{3} \quad \text { and } \quad H_{K}\left(K_{n}, K_{m}\right)<\frac{\varepsilon}{3} .
$$

This implies that $\left\{F_{n}(x)\right\}$ is a Cauchy sequence in $K(Y)$ and $\left\{K_{n}\right\}$ is a Cauchy sequence in $K(X)$. By the assumption and Lemma $2.5,\left(K(Y), H_{F}\right)$ and $\left(K(X), H_{K}\right)$ are complete. It follows that there exist $F(x) \in K(Y)$ and $K \in K(X)$ such that

$$
F_{n}(x) \rightarrow F(x) \quad \text { and } \quad K_{n} \rightarrow K
$$

For fixed $n \geq N_{1}$ and any $x \in K$, let $m \rightarrow+\infty$ in (1), we have

$$
H_{F}\left(F_{n}(x), F(x)\right)<\frac{\varepsilon}{3} \quad \text { and } \quad H_{K}\left(K_{n}, K\right)<\frac{\varepsilon}{3} .
$$

We now show that $F$ is continuous. In fact, by the continuity of $F_{n}$ and Lemma 2.6, there exist a neighborhood $U\left(x_{0}\right)$ of $x_{0}$ and a positive integer $N_{2}$ such that

$$
H_{F}\left(F_{n}(x), F_{n}\left(x_{0}\right)\right)<\frac{\varepsilon}{3} \quad \text { for all } x \in U\left(x_{0}\right) \cap K, \text { for any } n \geq N_{2} .
$$

Let $N=\max \left\{N_{1}, N_{2}\right\}$. Combining with (2), (3) and (4) yields

$$
H_{F}\left(F(x), F\left(x_{0}\right)\right) \leq H_{F}\left(F(x), F_{n}(x)\right)+H_{F}\left(F_{n}(x), F_{n}\left(x_{0}\right)\right)+H_{F}\left(F_{n}\left(x_{0}\right), F\left(x_{0}\right)\right)<\varepsilon
$$


for all $x \in U\left(x_{0}\right) \cap K$ and for any $n \geq N$. By Lemma 2.6, $F$ is continuous on $K$. Set $u=(F, K)$ and so $u \in M$. It follows that

$$
\rho\left(u_{n}, u\right)=\sup _{x \in K} H_{F}\left(F_{n}(x), F(x)\right)+H_{K}\left(K_{n}, K\right)<\varepsilon
$$

which implies $u_{n} \stackrel{\rho}{\rightarrow} u$. Therefore, $(M, \rho)$ is a complete metric space. The proof is complete.

For any $u=(F, K) \in M$, we denote by $S(u)$ and $S_{w}(u)$ the efficient solution set and the weakly efficient solution set of problem (SOP), respectively. Then $S$ and $S_{w}$ define two set-valued maps from $M$ to $X$. By the compactness of $K$ and the continuity of $F$, the set $\operatorname{Min}(F(X))$ is nonempty, and so $S(u)$ is nonempty for any $u \in M$. Moreover, $S_{w}(u)$ is nonempty since $S(u) \subset S_{w}(u)$.

Theorem 3.1 The set-valued map $S_{w}: M \rightarrow 2^{X}$ is upper semicontinuous with compact values.

Proof For any $u=(F, K) \in M$, we prove that the set

$$
S_{w}(u)=\{x \in K:(F(K)-y) \cap-\operatorname{int} C=\emptyset, \exists y \in F(x)\}
$$

is compact. In fact, let $\left\{x_{n}\right\} \subseteq S_{w}(u)$ with $x_{n} \rightarrow x_{0}$. Then $x_{n} \in K$ and there exists $y_{n} \in F\left(x_{n}\right)$ such that

$$
\left(F(K)-y_{n}\right) \cap-\operatorname{int} C=\emptyset .
$$

Note that $K$ is a compact set. It follows that $x_{0} \in K$. Since $F(K) \supset F\left(x_{n}\right)$ is compact, there exists a subsequence of $\left\{y_{n}\right\}$ which converges to $y_{0}$. Without loss of generality, we may assume that $y_{n} \rightarrow y_{0}$. By the continuity of $F, y_{0} \in F\left(x_{0}\right)$. This fact together with (5) yields $x_{0} \in S_{w}(u)$. It follows that $S_{w}(u)$ is closed. Therefore, $S_{w}(u)$ is compact since $K$ is compact.

Next, we prove that $S_{w}$ is upper semicontinuous on $M$. Suppose by contradiction that there exists $u=(F, K) \in M$ such that $S_{w}$ is not upper semicontinuous at $u$. Then there exists an open neighborhood $U$ in $X$ with $U \supset S_{w}(u)$ such that, for each $n=1,2, \ldots$ and each open neighborhood $V_{n}:=\left\{u^{\prime}=\left(F^{\prime}, K^{\prime}\right) \in M: \rho\left(u^{\prime}, u\right)<\frac{1}{n}\right\}$ of $u$, there exist $u_{n}=\left(F_{n}, K_{n}\right) \in V_{n}$ and $x_{n} \in S_{w}\left(u_{n}\right)$ but $x_{n} \notin U$.

From $u_{n}=\left(F_{n}, K_{n}\right) \in V_{n}$ for each $n=1,2, \ldots$, we have $\rho\left(u_{n}, u\right)<\frac{1}{n} \rightarrow 0$. This implies

$$
F_{n} \rightarrow F \quad \text { and } \quad K_{n} \rightarrow K
$$

As $x_{n} \in S_{w}\left(u_{n}\right)$, we have $x_{n} \in K_{n}$ and there exists $y_{n} \in F_{n}\left(x_{n}\right)$ such that

$$
\left(F_{n}\left(K_{n}\right)-y_{n}\right) \cap-\operatorname{int} C=\emptyset .
$$

By the compactness of $K$ and $K_{n}$ and Lemma 2.2(i), $\bigcup_{n=1}^{+\infty} K_{n} \cup K$ is compact. Note that $\left\{x_{n}\right\} \subseteq \bigcup_{n=1}^{+\infty} K_{n} \cup K$. Then $\left\{x_{n}\right\}$ has a convergent subsequence. Without loss of generality, we may assume that $\left\{x_{n}\right\}$ is convergent. By Lemma 2.2(ii) and the uniqueness of the limit 
of $\left\{x_{n}\right\}, x_{n} \rightarrow x^{*} \in K$. Since $x_{n} \notin U$ and $U$ is open, $x^{*} \notin U$. From $S_{w}(u) \subset U$, we have $x^{*} \notin$ $S_{w}(u)$. It follows that

$$
(F(K)-y) \cap-\operatorname{int} C \neq \emptyset, \quad \forall y \in F\left(x^{*}\right) .
$$

On the other hand, since $y_{n} \in F_{n}\left(x_{n}\right)$ and $F_{n}\left(x_{n}\right)$ is compact for any $n$, there exists $y_{0}$ such that $y_{n} \rightarrow y_{0}$. By Lemma 2.7, $y_{0} \in F\left(x^{*}\right)$. Note that $K_{n} \rightarrow K$. Then, for any $z \in K$, there exists a sequence $\left\{z_{n}\right\}$ such that $z_{n} \in K_{n}$ and $z_{n} \rightarrow z$. By Lemma 2.8, for any $w \in F(z)$, there exists $w_{n} \in F_{n}\left(z_{n}\right)$ such that $w_{n} \rightarrow w$. Since $\left(F_{n}\left(K_{n}\right)-y_{n}\right) \cap-\operatorname{int} C=\emptyset$, one has

$$
w_{n}-y_{n} \notin-\operatorname{int} C \text {. }
$$

It follows that

$$
w-y_{0} \notin-\operatorname{int} C \text {. }
$$

This contradicts (6). Therefore, $S_{w}$ is upper semicontinuous on $M$. The proof is complete.

From the proof of Theorem 3.1, we obtain that for any $u \in M$, the weakly efficient solution set $S_{w}(u)$ is closed. By Lemma 2.1, we have the following corollary.

Corollary 3.1 The set-valued map $S_{w}: M \rightarrow 2^{X}$ is closed.

Remark 3.1 Corollary 3.1 generalizes and improves the corresponding result of Song et al. [29], Theorem 3.1, in the following four aspects:

(1) the assumption that the metric space is compact is removed;

(2) the setting of Euclidean spaces is generalized to Banach spaces;

(3) the order cone $\mathbb{R}_{+}^{n}$ is generalized to any closed convex pointed cone;

(4) we not only consider the perturbation of the set-valued map, but also consider the perturbation of the feasible set; while Song et al. [29] only considered the former.

Definition 3.1 Let $u \in M$. The weakly efficient solution set $S_{w}(u)$ is called stable if the set-valued map $S_{w}$ is continuous at $u$.

Remark 3.2 The following example shows that there exists $u \in M$ such that $S_{w}(u)$ is not stable.

Example 3.1 Let $X=\mathbb{R}, Y=\mathbb{R}^{2}, C=\mathbb{R}_{+}^{2}, K=[0,1]$ and $K_{n}=\left[\frac{1}{n}, 1\right]$. Define set-valued mappings $F, F_{n}: X \rightarrow 2^{\mathbb{R}^{2}}$ such that for any $x \in X$,

$$
F(x)=[0,1] \times[x, 1] \quad \text { and } \quad F_{n}(x)=\left[\frac{x}{n}, 1\right] \times[x, 1] .
$$

Then $F_{n} \rightarrow F$ and $K_{n} \rightarrow K$ when $n \rightarrow+\infty$. By a simple computation,

$$
S_{w}(u)=[0,1], \quad u=(F, K),
$$




$$
S_{w}\left(u_{n}\right)=\frac{1}{n}, \quad u_{n}=\left(F_{n}, K_{n}\right)
$$

It is easy to see that $S_{w}$ is upper semicontinuous at $u$. However, $S_{w}$ is not lower semicontinuous at $u$. In fact, let $x_{0}=1 \in S_{w}(u)$, one can easily find that for small enough neighborhood $U\left(x_{0}\right)$ of $x_{0}$ and large enough $n, S_{w}\left(u_{n}\right) \cap U\left(x_{0}\right)=\emptyset$. Therefore, $S_{w}$ is not stable at $u$.

Definition 3.2 For $u \in M$, a point $x \in S_{w}(u)$ is said to be essential if, for any open neighborhood $U$ of $x$ in $X$, there exists an open neighborhood $V$ of $u$ in $M$ such that $S_{w}\left(u^{\prime}\right) \cap U \neq \emptyset$ for all $u^{\prime} \in V . u$ is said to be essential if every $x \in S_{w}(u)$ is essential.

From Definition 3.2, it is easy to see that the following lemma holds, so we omit its proof.

Lemma 3.2 The set-valued map $S_{w}$ is lower semicontinuous at $u \in M$ if and only if $u$ is essential.

We now give a generic stability result for set-valued optimization problems.

Theorem 3.2 There exists a dense residual subset $Q$ of $M$ such that, for every $u \in Q, u$ is essential.

Proof By Lemmas 3.1 and 2.3, $M$ is a Baire space. By Theorem 3.1, the set-valued map $S_{w}$ : $M \rightarrow 2^{X}$ is upper semicontinuous with compact values. By Lemma 2.4, there exists a dense residual subset $Q$ of $M$ such that $S_{w}$ is lower semicontinuous at each $u \in Q$. Therefore, the conclusion holds by Lemma 3.2.

Remark 3.3 Example 3.1 shows that there exists $u \in M$ such that $u$ is not essential.

The following theorem gives a sufficient condition that $u \in M$ is essential.

Theorem 3.3 If $u \in M$ and $S_{w}(u)$ is a singleton set, then $u$ is essential.

Proof Suppose that $S_{w}(u)=\left\{x_{0}\right\}$. Let $U$ be any open set in $X$ such that $S_{w}(u) \cap U \neq \emptyset$. Then $x_{0} \in U$ and $S_{w}(u) \subset U$. By Theorem 3.1, $S_{w}$ is upper semicontinuous at $u \in M$. It follows that there exists an open neighborhood $V$ of $u$ in $M$ such that $S_{w}\left(u^{\prime}\right) \subset U$ for each $u^{\prime} \in V$. This implies that $S_{w}\left(u^{\prime}\right) \cap U \neq \emptyset$ for each $u^{\prime} \in V$. Thus, $S_{w}$ is lower semicontinuous at $u$. By Lemma 3.2, $u$ is essential.

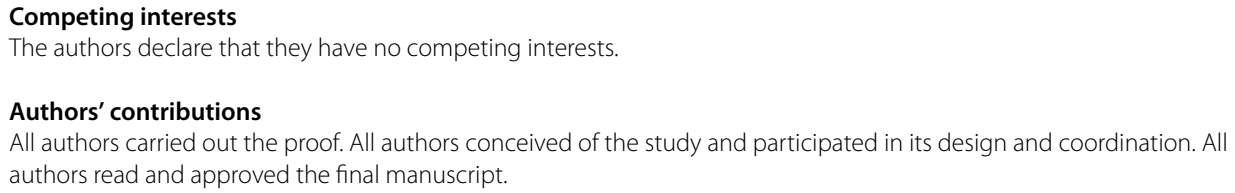

\section{Acknowledgements}

The first author was supported by the National Natural Science Foundation of China $(11001287,11471059)$, the Chongqing Research Program of Basic Research and Frontier Technology (cstc2014jcyjA00037), the Education Committee Project Research Foundation of Chongqing (KJ1400618) and the Program for Core Young Teacher of the Municipal Higher Education of Chongqing ([2014]47). 


\section{References}

1. Ansari, QH, Jahn, J: T-Epiderivative of set-valued maps and its application to set optimization and generalized variational inequalities. Taiwan. J. Math. 14, 2447-2468 (2010)

2. Aubin, JP, Frankowska, H: Set-Valued Analysis. Birkhäuser, Boston (1990)

3. Chen, GY, Jahn, J: Optimality conditions for set-valued optimization problems. Math. Methods Oper. Res. 48, 187-200 (1998)

4. Chen, GY, Huang, XX, Yang, XQ: Vector Optimization: Set-Valued and Variational Analysis. Springer, Berlin (2005)

5. Durea, $M$, Strugariu, R: Calculus of tangent sets and derivatives of set-valued maps under metric subregularity conditions. J. Glob. Optim. 56, 587-603 (2013)

6. Flores-Bazán, F: Radial epiderivatives and asymptotic functions in nonconvex vector optimization. SIAM J. Optim. 14, 284-305 (2003)

7. Gong, XH, Dong, HB, Wang, SY: Optimality conditions for proper efficient solutions of vector set-valued optimization. J. Math. Anal. Appl. 284, 332-350 (2003)

8. Hernández, E, Rodríguez-Marín, L, Sama, M: On solutions of set-valued optimization problems. Comput. Math. Appl. 60, 1401-1408 (2010)

9. Jahn, J: Vector Optimization: Theory, Applications, and Extensions. Springer, Berlin (2004)

10. Kuroiwa, D: On derivatives of set-valued maps and optimality conditions for set optimization. J. Nonlinear Convex Anal. 10, 41-50 (2009)

11. Khan, AA, Tammer, C, Zalinescu, C: Set-Valued Optimization: An Introduction with Applications. Springer, Berlin (2015)

12. Lalitha, CS, Arora, R: Weak Clarke epiderivative in set-valued optimization. J. Math. Anal. Appl. 342, 704-714 (2008)

13. Luc, DT: Theory of Vector Optimization. Lecture Notes in Economics and Mathematics Systems, vol. 319. Springer New York (1989)

14. Long, XJ, Li, XB, Zeng, J: Lagrangian conditions for approximate solutions on nonconvex set-valued optimization problems. Optim. Lett. 7, 1847-1856 (2013)

15. Long, XJ, Peng, JW: Lagrangian duality for vector optimization problems with set-valued mappings. Taiwan. J. Math. $17,287-297(2013)$

16. Long, XJ, Peng, JW: Generalized B-well-posedness for set optimization problems. J. Optim. Theory Appl. 157, 612-623 (2013)

17. Long, XJ, Peng, JW, Li, XB: Weak subdifferentials for set-valued mappings. J. Optim. Theory Appl. 162, 1-12 (2014)

18. Long, XJ, Peng, JW, Peng, ZY: Scalarization and pointwise well-posedness for set optimization problems. J. Glob. Optim. 62, 763-773 (2015)

19. Tammer, C, Zalinescu, C: Vector variational principles for set-valued functions. In: Recent Developments in Vector Optimization, pp. 367-415. Springer, Berlin (2012)

20. Qiu, QS, Yang, XM: Some properties of approximate solutions for vector optimization problem with set-valued functions. J. Glob. Optim. 47, 1-12 (2010)

21. Zhou, ZA, Yang, XM: Scalarization of $\varepsilon$-super efficient solutions of set-valued optimization problems in real ordered linear spaces. J. Optim. Theory Appl. 162,680-693 (2014)

22. Fort, MK: Essential and nonessential fixed points. Am. J. Math. 72, 315-322 (1950)

23. Yu, J, Xiang, SW: The stability of the set of KKM points. Nonlinear Anal. 54, 839-844 (2003)

24. Chen, JC, Gong, XH: The stability of set of solutions for symmetric vector quasi-equilibrium problems. J. Optim. Theory Appl. 136, 359-374 (2008)

25. Yang, H, Yu, J: Essential components of the set of weakly Pareto-Nash equilibrium points. Appl. Math. Lett. 15, 553-560 (2002)

26. Lin, Z: Essential components of the set of weakly Pareto-Nash equilibrium points for multiobjective generalized games in different topological spaces. J. Optim. Theory Appl. 124, 387-450 (2005)

27. Luo, Q: Essential component and essential optimum solution of optimization problems. J. Optim. Theory Appl. 102, 433-438 (1999)

28. Xiang, SW, Zhou, YH: On essential sets and essential components of efficient solutions for vector optimization problems. J. Math. Anal. Appl. 315, 317-326 (2006)

29. Song, QQ, Tang, GQ, Wang, LS: On essential stable sets of solutions in set optimization problems. J. Optim. Theory Appl. 156, 591-599 (2013)

30. Klein, E, Thompson, AC: Theory of Correspondences. Wiley, New York (1968)

31. Kuratowski, K: Topology, Vols. 1 and 2. Academic Press, New York (1968)

32. Yu, J: Essential weak efficient solution in multiobjective optimization problems. J. Math. Anal. Appl. 166, 230-235 (1992) 